R. Todor, I. Chiose and G. Marinescu

Nagoya Math. J.

Vol. 163 (2001), 145-165

\title{
MORSE INEQUALITIES FOR COVERING MANIFOLDS
}

\author{
RADU TODOR, IONUŢ CHIOSE AND GEORGE MARINESCU ${ }^{1}$
}

\begin{abstract}
We study the existence of $L^{2}$ holomorphic sections of invariant line bundles over Galois coverings. We show that the von Neumann dimension of the space of $L^{2}$ holomorphic sections is bounded below under weak curvature conditions. We also give criteria for a compact complex space with isolated singularities and some related strongly pseudoconcave manifolds to be Moishezon. As applications we prove the stability of the previous Moishezon pseudoconcave manifolds under perturbation of complex structures as well as weak Lefschetz theorems.
\end{abstract}

In this paper we wish to address the following problem. Let $\widetilde{M}$ be a complex manifold and assume there is a discrete group $\Gamma \subset \operatorname{Aut}(\widetilde{M})$ acting freely and properly discontinuously on $\widetilde{M}$. Suppose that $E \longrightarrow M$ is a holomorphic line bundle on $M=\widetilde{M} / \Gamma$. We denote by $\pi: \widetilde{M} \longrightarrow M$ the canonical projection.

Problem. Find non-trivial $L^{2}$ holomorphic sections in $\pi^{*} E^{k}$ over $\widetilde{M}$ provided $E$ satisfies reasonable conditions in terms of curvature positivity.

Gromov, Henkin and Shubin [12, Theorem 0.2] showed that, if $\bar{M}$ is a strongly pseudoconvex domain, the von Neumann $\Gamma$-dimension (see Atiyah $[5, \S 3]$ ) of the space of holomorphic $L^{2}$ functions on $\widetilde{M}$ (with respect to a $\Gamma-$ invariant metric) is infinite. Our aim is to generalize in a similar manner the following Morse inequality of Siu-Demailly [24], [9]. Namely, assume that $M$ is a compact manifold, $E \longrightarrow M$ is a semi-positive holomorphic line bundle which is positive at one point. Then $\operatorname{dim} H^{0}\left(M, E^{k}\right) \gtrsim k^{n}$, for large $k$, where $n=\operatorname{dim} M$. To this end we use the tools of [26] in which Shubin gives a proof in the spirit of Witten [28] of the Morse inequalities for covering manifolds (so called Novikov-Shubin inequalities). In $\S 1$ we generalize the Weyl type formula of Demailly by describing the asymptotic behaviour of the spectrum

Received January 24, 2000.

2000 Mathematics Subject Classification: 58J37, 32F10, 32 Q55.

${ }^{1}$ Supported by a DFG Stipendium at the Graduiertenkolleg 'Geometrie und Nichtlineare Analysis' at Humboldt-Universität zu Berlin and SFB 288. 
of a $\Gamma$-invariant laplacian associated to high powers of a $\Gamma$-invariant line bundle. We give in $\S 2$, Theorem 2.1, a general answer to our problem. We focus in $\S 3$ on the case of pseudoconcave manifolds and establish criteria for some classes of 1-concave manifolds to be Moishezon. We prove the stability of some Moishezon strongly pseudoconcave manifolds under perturbation of complex structures. As application of Theorem 2.1 we prove in $\S 4$ weak Lefschetz theorems using the analytic proof (and generalization) of Nori's results due to Napier and Ramachandran [19].

\section{$\S 1$. Estimates of the spectrum distribution function}

As before let $M$ be a complex manifold of dimension $n$ and $\pi: \widetilde{M} \longrightarrow M$ a normal covering of group $\Gamma$. We assume $\widetilde{M}$ paracompact so that $\Gamma$ will be countable. Suppose we are given a holomorphic vector bundle $F$ on $M$ and take its pull-back $\widetilde{F}=\pi^{*} F$, which is a $\Gamma$-invariant bundle on $\widetilde{M}$. We also fix a $\Gamma$-invariant hermitian metric on $\widetilde{M}$ and on $\widetilde{F}$. We consider an open set $\Omega \Subset M$ with smooth boundary and its preimage $\widetilde{\Omega}=\pi^{-1} \Omega ; \Gamma$ acts on $\widetilde{\Omega}$ and $\widetilde{\Omega} / \Gamma=\Omega$. In general we will decorate with tildes the preimages of objects living on the quotient. Let $U$ be a fundamental domain of the action of $\Gamma$ on $\widetilde{\Omega}$. This means that (see e.g. [5, p.52]): (a) $\widetilde{\Omega}$ is covered by the translations of $\bar{U}$, (b) different translations of $U$ have empty intersection and (c) $\bar{U} \backslash U$ has zero measure (since $\partial \Omega$ is smooth). $\Omega$ being relatively compact $U$ has the same property. Let us define the space of square integrable sections $L^{2}(\widetilde{\Omega}, \widetilde{F})$ with respect to a $\Gamma$-invariant metric on $\widetilde{M}$ (and its volume form) and a $\Gamma$-invariant metric on $\widetilde{F}$. Then $L^{2}(U, \widetilde{F})$ is constructed with respect to the same. There is a unitary action of $\Gamma$ on $L^{2}(\widetilde{\Omega}, \widetilde{F})$. In fact it is easy to see that $L^{2}(\widetilde{\Omega}, \widetilde{F}) \cong L^{2} \Gamma \otimes L^{2}(U, \widetilde{F}) \cong L^{2} \Gamma \otimes L^{2}(\Omega, F)$. We have a unitary action of $\Gamma$ on $L^{2} \Gamma$ by left translations: $l_{\gamma} \delta_{\alpha}=\delta_{\gamma \alpha}$ where $\left\{\delta_{\alpha}: \alpha \in \Gamma\right\}$ is the orthonormal basis of $L^{2} \Gamma$ formed by the delta functions. It induces an action on $L^{2}(\widetilde{\Omega}, \widetilde{F})$ by $\gamma \longmapsto L_{\gamma}=l_{\gamma} \otimes$ Id. Finally we denote by $\mathcal{D}(.,$.$) the$ various spaces of smooth compactly supported sections.

Let us consider a formally self-adjoint, strongly elliptic, positive differential operator $P$ on $M$ acting on sections of $F$. Denote by $\widetilde{P}$ the $\Gamma$-invariant differential operator which is its pull-back to $\widetilde{M}$. From $\widetilde{P}$ we construct the following operators: the Friedrichs extension in $L^{2}(\widetilde{\Omega}, \widetilde{F})$ of $\widetilde{P}$ with domain $\mathcal{D}(\widetilde{\Omega}, \widetilde{F})$ and the Friedrichs extension in $L^{2}(U, \widetilde{F})$ of $\widetilde{P}$ with domain $\mathcal{D}(U, \widetilde{F})$. From now on we denote these extensions by $\widetilde{P}$ and $P_{U}$. They are closed self-adjoint positive operators. It is known that $\widetilde{P}$ is also $\Gamma$-invariant i.e. it commutes with all $L_{\gamma}$. This amounts to saying that the spectral projectors 
$E(\lambda, \widetilde{P})$ of $\widetilde{P}$ commute with all $L_{\gamma}$. On the other hand the Rellich lemma tells us that $P_{U}$ has compact resolvent and hence discrete spectrum. We will undertake the task of comparing the distribution of the two spectra. Namely since $E(\lambda, \widetilde{P})$ is $\Gamma$-invariant its image $R(E(\lambda, \widetilde{P}))$ is a $\Gamma$-invariant closed subspace of the free Hilbert $\Gamma$-module $L^{2} \Gamma \otimes L^{2}(U, \widetilde{F}) \cong L^{2}(\widetilde{\Omega}, \widetilde{F})$. In general for any Hilbert space $\mathcal{H}$ we call the Hilbert space $L^{2} \Gamma \otimes \mathcal{H}$ a free Hilbert $\Gamma$-module. The action of $\Gamma$ is defined as above by $\gamma \longmapsto L_{\gamma}=l_{\gamma} \otimes I d$. To any $\Gamma$-invariant closed space (called a $\Gamma$-module) one can associate a positive, possibly infinite real number, called von Neumann or $\Gamma$-dimension, denoted $\operatorname{dim}_{\Gamma}$. For notions involving the $\Gamma$-dimension and linear algebra for $\Gamma$-modules we refer the reader to [5], [26] and [14, pp.75-80]. Let us just remark that if $L \subset L^{2}(\widetilde{\Omega}, \widetilde{F})$ is a $\Gamma$-module and $f_{i}$ is an orthonormal basis of $L$,

$$
\operatorname{dim}_{\Gamma} L=\sum_{i} \int_{U}\left|f_{i}\right|^{2} .
$$

We denote in the sequel $N_{\Gamma}(\lambda, \widetilde{P})=\operatorname{dim}_{\Gamma} R(E(\lambda, \widetilde{P}))$. Similarly we consider the counting function of $P_{U}, N\left(\lambda, P_{U}\right)=\operatorname{dim} R\left(E\left(\lambda, P_{U}\right)\right)$. In order to compare $N_{\Gamma}(\lambda, \widetilde{P})$ and $N\left(\lambda, P_{U}\right)$ we use essentially the analysis of Shubin [26]. Let $\widetilde{P}$ be a $\Gamma$-invariant self-adjoint positive operator on a free $\Gamma$-module $L^{2} \Gamma \otimes \mathcal{H}$ where $\mathcal{H}$ is Hilbert space. Then we have the following variational principle [26, Lemma 2.4]:

(1.2) $N_{\Gamma}(\lambda, \widetilde{P})=\sup \left\{\operatorname{dim}_{\Gamma} L \mid L \subset \operatorname{Dom}(\widetilde{Q}), \widetilde{Q}(f, f) \leqslant \lambda\|f\|^{2}, \forall f \in L\right\}$

where $L$ is a $\Gamma$-module and $\widetilde{Q}$ is the quadratic form of $\widetilde{P}$.

Proposition 1.1. (Estimate from below) For all $\lambda \in \mathbb{R}$,

$$
N_{\Gamma}(\lambda, \widetilde{P}) \geqslant N\left(\lambda, P_{U}\right) .
$$

Proof. Let us denote by $\lambda_{0} \leqslant \lambda_{1} \leqslant \ldots$ the spectrum of $P_{U}$. Let $\left\{e_{i}\right\}$ be an orthonormal basis of $L^{2}(U, \widetilde{F})$ which consists of eigenfunctions of $P_{U}$ corresponding to the eigenvalues $\lambda_{i}$. Let $\widetilde{e}_{i}$ be the extension by 0 on $\widetilde{\Omega} \backslash \bar{U}$ of $e_{i}$. Then $\left\{L_{\gamma} \widetilde{e}_{i}\right\}$ is an orthonormal basis of $L^{2}(\widetilde{\Omega}, \widetilde{F})$ and $L_{\gamma} \widetilde{e}_{i} \in \operatorname{Dom}(\widetilde{Q})$. Let $\Phi_{\lambda}$ the $\Gamma$-module spanned by the orthonormal set $\left\{L_{\gamma} \widetilde{e}_{i}: \lambda_{i} \leqslant \lambda\right\}$ in $L^{2}(\widetilde{\Omega}, \widetilde{F})$. Then by $(1.1) \operatorname{dim}_{\Gamma} \Phi_{\lambda}=\sum_{\lambda_{i} \leqslant \lambda} 1=N\left(\lambda, P_{U}\right)$. Moreover, it is easy to see that $\Phi_{\lambda} \subset \operatorname{Dom}(\widetilde{Q})$ and $\widetilde{Q}(f, f) \leqslant \lambda\|f\|^{2}, f \in \Phi_{\lambda}$, as $\operatorname{Dom}(\widetilde{Q})$ is complete in the graph norm. Thus (1.3) follows from (1.2). 
The next step is an estimate from above of $N_{\Gamma}(\lambda, \widetilde{P})$. Let $L_{1}, L_{2}$ be two $\Gamma$-modules. A bounded linear operator $T: L_{1} \longrightarrow L_{2}$ is called a $\Gamma-$ morphism if it commutes with the action of $\Gamma$. By [26, p. 398], if $T$ is injective, $\operatorname{dim}_{\Gamma} L_{1} \leqslant \operatorname{dim}_{\Gamma} L_{2}$ and if $T$ has dense image, $\operatorname{dim}_{\Gamma} L_{1} \geqslant \operatorname{dim}_{\Gamma} L_{2}$. Set $\operatorname{rank}_{\Gamma} T=\operatorname{dim}_{\Gamma} \overline{R(T)}$. For the following we refer to [26, Lemma 3.7].

LEMMA 1.2. Let us consider the same setting as in the variational principle. Assume $T: L^{2}(\widetilde{\Omega}, \widetilde{F}) \rightarrow L^{2}(\widetilde{\Omega}, \widetilde{F})$ is a $\Gamma$-morphism such that $((\widetilde{P}+T) f, f) \geqslant \mu\|f\|^{2}, f \in \operatorname{Dom}(\widetilde{P})$ and $\operatorname{rank}_{\Gamma} T \leqslant p$. Then

$$
N_{\Gamma}(\mu-\varepsilon, \widetilde{P}) \leqslant p, \quad \forall \varepsilon>0 .
$$

In order to get an estimate from above we have to enlarge a little bit the fundamental domain $U$ and compare the counting function of $\widetilde{P}$ on $\widetilde{\Omega}$ to the counting function of $\widetilde{P}$ with Dirichlet boundary conditions on the enlarged domain. For $h>0$, let $U_{h}=\{x \in \widetilde{\Omega}: d(x, U)<h\}$ where $d$ is the distance on $\widetilde{M}$ associated to the Riemann metric on $\widetilde{M}$ and then let $U_{h, \gamma}:=\gamma U_{h}$. Next we need a partition of unity. Let $\varphi^{(h)} \in C^{\infty}(\widetilde{\Omega}), \varphi^{(h)} \geqslant 0$, $\varphi^{(h)}=1$ on $\bar{U}$ and $\operatorname{supp} \varphi^{(h)} \subset U_{h}, \varphi_{\gamma}^{(h)}=\varphi^{(h)} \circ \gamma^{-1}$. We define the function $J_{\gamma}^{(h)} \in C^{\infty}(\widetilde{\Omega})$ by $J_{\gamma}^{(h)}=\varphi_{\gamma}^{(h)}\left(\sum_{\gamma}\left(\varphi_{\gamma}^{(h)}\right)^{2}\right)^{-\frac{1}{2}}$ so that $\sum_{\gamma \in \Gamma}\left(J_{\gamma}^{(h)}\right)^{2}=1$. If $\widetilde{P}$ is of order 2 , which will be assumed from now on, by [26, Lemma 3.1] (variant of IMS localization formula, see [8]),

$$
\widetilde{P}=\sum_{\gamma \in \Gamma} J_{\gamma}^{(h)} \widetilde{P} J_{\gamma}^{(h)}-\sum_{\gamma \in \Gamma} \sigma_{0}(\widetilde{P})\left(d J_{\gamma}^{(h)}\right)
$$

where $\sigma_{0}$ is the principal symbol of $\widetilde{P}$. Since the derivative of $J_{\gamma}^{(h)}$ is $O\left(h^{-1}\right)$ and the order of $\widetilde{P}$ is 2 we deduce that there exists $C>0$ independent of $h$ such that:

$$
\widetilde{P} \geqslant \sum_{\gamma \in \Gamma} J_{\gamma}^{(h)} \widetilde{P} J_{\gamma}^{(h)}-\frac{C}{h^{2}} \mathrm{Id}
$$

We let $\widetilde{P}$ act on $\mathcal{D}\left(U_{h, \gamma}, \widetilde{F}\right)$ and take its Friedrichs extension $P_{U_{h, \gamma}}$. Since $P_{U_{h, \gamma}}$ is positive, $P_{U_{h, \gamma}}+\lambda E\left(\lambda, P_{U_{h, \gamma}}\right) \geqslant \lambda \mathrm{Id}$. We define the bounded operators $G_{\gamma}$ on $L^{2}(\widetilde{\Omega}, \widetilde{F})$ given by $G_{\gamma}=J_{\gamma}^{(h)} \lambda E\left(\lambda, P_{U_{h, \gamma}}\right) J_{\gamma}^{(h)}$ and $G=$ $\sum_{\gamma \in \Gamma} G_{\gamma}$. Since $J_{\gamma}^{(h)} \widetilde{P} J_{\gamma}^{(h)}=J_{\gamma}^{(h)} P_{U_{h, \gamma}} J_{\gamma}^{(h)},(1.5)$ yields

$$
\widetilde{P}+G \geqslant\left(\lambda-\frac{C}{h^{2}}\right) \mathrm{Id}
$$




\section{Claim 1.3.}

$$
\operatorname{rank}_{\Gamma} G \leqslant N\left(\lambda, P_{U_{h}}\right)
$$

Proof. We start with the finite rank operator $\bar{G}$ on $L^{2}\left(U_{h}, \widetilde{F}\right), \bar{G}=$ $J_{e}^{(h)} \lambda E\left(\lambda, P_{U_{h}}\right) J_{e}^{(h)}$. Then, $\operatorname{rank} \bar{G} \leqslant \operatorname{rank} E\left(\lambda, P_{U_{h}}\right)=N\left(\lambda, P_{U_{h}}\right)$. Next we consider the free $\Gamma$-module $L^{2} \Gamma \otimes L^{2}\left(U_{h}, \widetilde{F}\right)$ and the bounded $\Gamma$-invariant operator $\operatorname{Id} \otimes \bar{G}$. Then $R(\operatorname{Id} \otimes \bar{G})=L^{2} \Gamma \otimes R(\bar{G})$ so that $\operatorname{rank}_{\Gamma} \operatorname{Id} \otimes \bar{G}=\operatorname{rank} \bar{G}$. We now identify the space $L^{2} \Gamma \otimes L^{2}\left(U_{h}, \widetilde{F}\right)$ with $\bigoplus_{\gamma \in \Gamma} L^{2}\left(U_{h, \gamma}, \widetilde{F}\right)$ by the unitary transform $K: \sum_{\gamma} \delta_{\gamma} \otimes w_{\gamma} \longmapsto\left(L_{\gamma} w_{\gamma}\right)_{\gamma}$. Thus $\bigoplus_{\gamma \in \Gamma} L^{2}\left(U_{h, \gamma}, \widetilde{F}\right)$ is naturally a free $\Gamma$-module for which $K$ is $\Gamma$-invariant. We transport Id $\otimes \bar{G}$ on $\bigoplus_{\gamma \in \Gamma} L^{2}\left(U_{h, \gamma}, \widetilde{F}\right)$ by $K$ and we think of it as acting on this latter space. We construct then a restriction operator $V: \bigoplus_{\gamma \in \Gamma} L^{2}\left(U_{h, \gamma}, \widetilde{F}\right) \longrightarrow$ $L^{2}(\widetilde{\Omega}, \widetilde{F}), V\left(\left(w_{\gamma}\right)_{\gamma}\right)=\sum_{\gamma \in \Gamma} w_{\gamma}$ which is a surjective $\Gamma$-morphism. We have also the $\Gamma$-morphism $I$ from $L^{2}(\widetilde{\Omega}, \widetilde{F})$ to $\bigoplus_{\gamma \in \Gamma} L^{2}\left(U_{h, \gamma}, \widetilde{F}\right), I(u)=$ $\left(u \uparrow_{U_{h, \gamma}}\right)_{\gamma}$ which is obviously bounded. With our identifications, and replacing $E\left(\lambda, P_{U_{\gamma, h}}\right)$ by $L_{\gamma} E\left(\lambda, P_{U_{h}}\right) L_{\gamma}^{-1}$ in the definition of $G_{\gamma}$, we have $G=V(\operatorname{Id} \otimes \bar{G}) I$. As in the case of usual dimension $\operatorname{rank}_{\Gamma} V(\operatorname{Id} \otimes \bar{G}) I \leqslant$ $\operatorname{rank}_{\Gamma}(\operatorname{Id} \otimes \bar{G})\left(\right.$ see $\left[26\right.$, Lemma 3.6]). Hence $\operatorname{rank}_{\Gamma} G \leqslant \operatorname{rank}_{\Gamma}(\operatorname{Id} \otimes \bar{G})=$ $\operatorname{rank} \bar{G} \leqslant N\left(\lambda, P_{U_{h}}\right)$.

Proposition 1.4. (Estimate from above) There is a constant $C>0$ such that

$$
N_{\Gamma}(\lambda, \widetilde{P}) \leqslant N\left(\lambda+\frac{C}{h^{2}}, P_{U_{h}}\right) \quad \lambda \in \mathbb{R}, \quad h>0 .
$$

Proof. We obtain $N_{\Gamma}(\lambda, \widetilde{P}) \leqslant N\left(\lambda+\frac{C}{h^{2}}+\varepsilon, P_{U_{h}}\right)$ by Lemma 1.2 , (1.6), (1.7) and let $\varepsilon \longrightarrow 0$ (the counting function is right continuous).

We are going to apply the above results to the semi-classical asymptotics as $k \longrightarrow \infty$ of the spectral distribution function of the laplacian $k^{-1} \widetilde{\Delta}_{k}^{\prime \prime}$ on $\widetilde{M}$. We let $\widetilde{E}$ and $\widetilde{G}$ be two $\Gamma$-invariant holomorphic line bundles. Let us form the Laplace-Beltrami operator $\widetilde{\Delta}_{k}^{\prime \prime}=\bar{\partial} \vartheta+\vartheta \bar{\partial}$ on $(0, q)$ forms with values in $\widetilde{E}^{k} \otimes \widetilde{G}$. We apply the previous results for $\widetilde{P}=k^{-1} \widetilde{\Delta}_{k}^{\prime \prime}\lceil\widetilde{\Omega}$, the operator of the Dirichlet problem on $\widetilde{\Omega}$. Now we have to make a good choice of the parameter $h$. We take $h=k^{-\frac{1}{4}}$ so that the derivative of the cutting off function $J_{\gamma}^{(h)}$ is just $O\left(k^{\frac{1}{4}}\right)$. Then $\sigma_{0}\left(k^{-1} \widetilde{\Delta}_{k}^{\prime \prime}\right)\left(d J_{\gamma}^{(h)}\right)=k^{-1}\left|\bar{\partial} J_{\gamma}^{(h)}\right|^{2}=$ $O\left(k^{-\frac{1}{2}}\right)$. Modifying (1.5), (1.6) and (1.8) accordingly we obtain the following semi-classical estimate. 
Proposition 1.5. There exists a constant $C>0$ independent of $k$ such that for $\lambda \in \mathbb{R}$ and $k>0$ we have

$$
\left.N\left(\lambda, \frac{1}{k} \widetilde{\Delta}_{k}^{\prime \prime}\right\rceil_{U}\right) \leqslant N_{\Gamma}\left(\lambda, \frac{1}{k} \widetilde{\Delta}_{k}^{\prime \prime} \Upsilon_{\Omega}\right) \leqslant N\left(\lambda+\frac{C}{\sqrt{k}}, \frac{1}{k} \widetilde{\Delta}_{k}^{\prime \prime} \Upsilon_{U_{k^{-1 / 4}}}\right)
$$

Demailly has determined the spectrum distribution for the Dirichlet problem for $k^{-1} \widetilde{\Delta}_{k}^{\prime \prime}$ in [9, Théorèmes 2.16, 3.14]. He introduces an integral $I^{q}(V, \lambda)$ over an open set $V \Subset \widetilde{M}$ of a function $\bar{\nu}_{E}(x, \lambda)$ on $\widetilde{M} \times \mathbb{R}$ depending on $\mathbf{c}(\widetilde{E})$ and $\lambda$, right continuous in $\lambda$ and bounded on compacts of $\widetilde{M}[9$, pp.197, 205].

Proposition 1.6. (Demailly) Assume $\partial V$ has measure zero and the laplacian acts on $(0, q)$ forms. Then $\lim \sup _{k} k^{-n} N\left(\lambda, \frac{1}{k} \widetilde{\Delta}_{k}^{\prime \prime} \uparrow_{V}\right) \leqslant I^{q}(V, \lambda)$. There exists an at most countable set $\mathcal{N} \subset \mathbb{R}$ such that for $\lambda \in \mathbb{R} \backslash \mathcal{N}$ the limit of the left-hand side expression exists and we have equality.

Let us fix $\varepsilon>0$. Then $N\left(\lambda+\frac{C}{\sqrt{k}}, \frac{1}{k} \widetilde{\Delta}^{\prime \prime}\right) \leqslant N\left(\lambda+\varepsilon, \frac{1}{k} \widetilde{\Delta}^{\prime \prime} \uparrow_{U_{\varepsilon}}\right)$ since for sufficiently large $k$ we have $U_{k^{-\frac{1}{4}}} \subset U_{\varepsilon}$. So $\lim \sup _{k} k^{-n} N_{\Gamma}\left(\lambda, \frac{1}{k} \widetilde{\Delta}_{k}^{\prime \prime} \Upsilon_{\Omega}\right) \leqslant$ $I^{q}\left(U_{\varepsilon}, \lambda+\varepsilon\right)$ by (1.9) ( $\partial U_{\varepsilon}$ is negligible for small $\left.\varepsilon\right)$. The use of dominated convergence to make $\varepsilon \longrightarrow 0$ in the last integral yields:

THEOREM 1.7. The spectral distribution function of $\frac{1}{k} \widetilde{\Delta}_{k}^{\prime \prime} \Gamma_{\widetilde{\Omega}}$ acting on $L_{0, q}^{2}\left(\widetilde{\Omega}, \widetilde{E}^{k} \otimes \widetilde{G}\right)$ with Dirichlet boundary values satisfies

$$
\limsup _{k} k^{-n} N_{\Gamma}\left(\lambda, \frac{1}{k} \widetilde{\Delta}_{k}^{\prime \prime} \Upsilon_{\Omega}\right) \leqslant I^{q}(U, \lambda) .
$$

Moreover, there exists an at most countable set $\mathcal{N} \subset \mathbb{R}$ such that for $\lambda$ in $\mathbb{R} \backslash \mathcal{N}$ the limit exists and we have equality in (1.10).

\section{$\S 2$. Geometric situations}

If $M$ is a complete Kähler manifold and $E$ a positive line bundle on $M$ the $L^{2}$ estimates of Andreotti-Vesentini-Hörmander allow us to find a lot of sections of $\widetilde{E}$ on a covering $\widetilde{M}$ (see e.g. [19]). We now prove:

THEOREM 2.1. Let $(M, \omega)$ be an $n$-dimensional complete hermitian manifold such that the torsion of $\omega$ is pointwise bounded and let $(E, h)$ be a holomorphic hermitian line bundle. Let $K \Subset M$ and a constant $C_{0}>0$ be such that $\imath \mathbf{c}(E, h) \geqslant C_{0} \omega$ on $M \backslash K$. Let $p: \widetilde{M} \longrightarrow M$ be a Galois covering 
with group $\Gamma, \widetilde{E}=p^{*} E$, and let $\Omega$ be any open set with smooth boundary, $K \Subset \Omega \Subset M$. Then, for $k \longrightarrow \infty$,

$$
\operatorname{dim}_{\Gamma} H_{(2)}^{n, 0}\left(\widetilde{M}, \widetilde{E}^{k}\right) \geqslant \frac{k^{n}}{n !} \int_{\Omega(\leqslant 1, h)}\left(\frac{\imath}{2 \pi} \mathbf{c}(E, h)\right)^{n}+o\left(k^{n}\right),
$$

where $H_{(2)}^{n, 0}\left(\widetilde{M}, \widetilde{E}^{k}\right)$ is the space of $(n, 0)$-forms with values in $\widetilde{E}^{k}$ which are $L^{2}$ with respect to any metric on $\widetilde{M}$ and the pullback of $h$ and $\Omega(\leqslant 1, h)$ is the subset of $\Omega$ where $\imath \mathbf{c}(E, h)$ is non-degenerate and has at most one negative eigenvalue.

Proof. We endow $\widetilde{M}$ with the metric $\widetilde{\omega}=p^{*} \omega$ and $\widetilde{E}$ with $\widetilde{h}=p^{*} h$. The operators $\bar{\partial}$ and Laplace-Beltrami are $\Gamma$-invariant. We remark that $\widetilde{G}$ being a $\Gamma$-invariant bundle on $\widetilde{M}, L^{2}(\widetilde{M}, \widetilde{G})$ is a free $\Gamma$-module (as in $\S 1$ ). It is standard to see that $\widetilde{\omega}$ is also complete. We take a fundamental domain $U$ for the action of $\Gamma$ on $\widetilde{\Omega}$ and put $\widetilde{K}=p^{-1} K$.

Since $p$ is locally biholomorphic we see that $\imath \mathbf{c}(\widetilde{E}, \widetilde{h}) \geqslant C_{0} \widetilde{\omega}$ on $\widetilde{M} \backslash \widetilde{K}$. Let $u$ be a smooth $(n, 1)$ form on $\widetilde{M}$ with values in $\widetilde{E}^{k}$ and compactly supported outside $\widetilde{\Omega}$. We apply now the Bochner-Kodaira-Nakano formula: $3\left(\widetilde{\Delta}_{k}^{\prime \prime} u, u\right) \geqslant 2\left(\left[\imath \mathbf{c}\left(\widetilde{E}^{k}\right), \widetilde{\Lambda}\right] u, u\right)-\left(\|\tau u\|^{2}+\|\bar{\tau} u\|^{2}+\left\|\tau^{*} u\right\|^{2}+\left\|\bar{\tau}^{*} u\right\|^{2}\right)$ where $\widetilde{\Lambda}$ is the interior product with $\widetilde{\omega}$ and the $\tau$ 's are the torsion operators of $\widetilde{\omega}(\tau=[\widetilde{\Lambda}, \partial \widetilde{\omega}])$. Therefore there exists a constant $C_{1}>0$ (depending just on the metric $\omega)$ such that $3\left(\widetilde{\Delta}_{k}^{\prime \prime} u, u\right) \geqslant 2 C_{0} k\|u\|^{2}-C_{1}\|u\|^{2}$, and hence

$$
\left(\widetilde{\Delta}_{k}^{\prime \prime} u, u\right) \geqslant \frac{C_{0} k}{2}\|u\|^{2}, \quad k \geqslant \frac{C_{1}}{2 C_{0}} .
$$

by the hypothesis on the curvature and torsion. Let $\rho \in \mathcal{C}^{\infty}(M)$ such that $\rho=0$ on $L$ and $\rho=1$ on $M \backslash \Omega$, where $L$ is a neighbourhood of $K$ in $\Omega$. We put $\widetilde{\rho}=\rho \circ p$. Let $u \in \mathcal{D}^{n, 1}\left(\widetilde{M}, \widetilde{E}^{k}\right)$, so that $\widetilde{\rho} u$ has support outside $\widetilde{K}$. We use now the elementary estimate:

$$
\left(\widetilde{\Delta}_{k}^{\prime \prime}(\widetilde{\rho} u), \widetilde{\rho} u\right) \leqslant \frac{3}{2}\left(\widetilde{\Delta}_{k}^{\prime \prime} u, u\right)+6 \sup |d \widetilde{\rho}|^{2}\|u\|^{2}
$$

Obviously $C_{2}=6 \sup |d \widetilde{\rho}|^{2}<\infty$. Estimates (2.2) and (2.3) yield

$$
\|u\|^{2} \leqslant \frac{12}{C_{0} k}\left(\widetilde{\Delta}_{k}^{\prime \prime} u, u\right)+4 \int_{\widetilde{\Omega}}|(1-\widetilde{\rho}) u|^{2}, \quad k \geqslant \frac{\max \left\{C_{1}, 16 C_{2}\right\}}{2 C_{0}}
$$


for any compactly supported $u$. Since the metric $\widetilde{\omega}$ is complete the density lemma of Andreotti and Vesentini [3] shows that $\widetilde{\Delta}_{k}^{\prime \prime}$ is essentially selfadjoint. Thus (2.4) is true for any $u$ in the domain of the quadratic form $\widetilde{Q}_{k}$ of the self-adjoint extension of $k^{-1} \widetilde{\Delta}_{k}^{\prime \prime}$. From relation (2.4) we infer that the spectral spaces corresponding to the lower part of the spectrum of $k^{-1} \widetilde{\Delta}_{k}^{\prime \prime}$ on $(n, 1)$-forms can be injected into the spectral spaces of the $\Gamma$-invariant operator $k^{-1} \widetilde{\Delta}_{k}^{\prime \prime}\lceil\widetilde{\Omega}$ which correspond to the Dirichlet problem on $\widetilde{\Omega}$ for $k^{-1} \widetilde{\Delta}_{k}^{\prime \prime}$. The latter operator was studied in $\S 1$. This idea appears in Witten's proof (see Henniart [13, Lemme 2.1]) and in Bouche [6, Théorème $2.1]$ in the context of $q$-convex manifolds in the sense of Andreotti-Grauert. We claim that for $\lambda<C_{0} / 24$,

$$
\begin{aligned}
& L_{k}^{1}(\lambda) \longrightarrow L_{k, \widetilde{\Omega}}^{1}\left(12 \lambda+C_{3} k^{-1}\right), \\
& u \longmapsto E\left(6 \lambda+C_{3} k^{-1}, k^{-1} \widetilde{\Delta}_{k}^{\prime \prime} \Upsilon_{\Omega}\right)(1-\widetilde{\rho}) u,
\end{aligned}
$$

is an injective $\Gamma$-morphism, where $L_{k}^{1}(\lambda)=R\left(E\left(\lambda, k^{-1} \widetilde{\Delta}_{k}^{\prime \prime}\right)\right)$ is the spectral space of $k^{-1} \widetilde{\Delta}_{k}^{\prime \prime}$ on $(n, 1)$-forms, $L_{k, \widetilde{\Omega}}^{1}(\mu)=R\left(E\left(\mu, k^{-1} \widetilde{\Delta}_{k}^{\prime \prime} \Upsilon_{\Omega}\right)\right)$ and $C_{3}=$ $8 C_{2}$. It is easy to see that $(2.5)$ is $\Gamma$-invariant. To prove the injectivity we choose $u \in L_{k}^{1}(\lambda), \lambda<C_{0} / 24$ to the effect that $\widetilde{Q}_{k}(u) \leqslant \lambda\|u\|^{2} \leqslant$ $\left(C_{0} / 24\right)\|u\|^{2}$. Plugging this relation in (2.4) we get

$$
\|u\|^{2} \leqslant 8 \int_{\widetilde{\Omega}}|(1-\widetilde{\rho}) u|^{2}, \quad u \in L_{k}^{1}(\lambda), \quad \lambda<C_{0} / 24 .
$$

Let us denote by $\widetilde{Q}_{k, \widetilde{\Omega}}$ the quadratic form of $k^{-1} \widetilde{\Delta}_{k}^{\prime \prime} \Upsilon_{\widetilde{\Omega}}$. Then by $(2.3)$ and $(2.6), \widetilde{Q}_{k, \widetilde{\Omega}}((1-\widetilde{\rho}) u) \leqslant \frac{3}{2} \widetilde{Q}_{k}(u)+\frac{C_{2}}{k}\|u\|^{2} \leqslant\left(12 \lambda+\frac{8 C_{2}}{k}\right) \int_{\widetilde{\Omega}}|(1-\widetilde{\rho}) u|^{2}$ which shows that if $E\left(12 \lambda+C_{3} k^{-1}, k^{-1} \widetilde{\Delta}_{k}^{\prime \prime}\left\lceil_{\widetilde{\Omega}}\right)(1-\widetilde{\rho}) u=0\right.$ then $(1-\widetilde{\rho}) u=0$ so that $u=0$ by (2.6). Therefore (2.5) is injective and hence

$$
N_{\Gamma}^{1}\left(\lambda, \frac{1}{k} \widetilde{\Delta}_{k}^{\prime \prime}\right) \leqslant N_{\Gamma}^{1}\left(12 \lambda+\frac{C_{3}}{k}, \widetilde{\Delta}_{k}^{\prime \prime} \Upsilon_{\Omega}\right), \quad \lambda<\left(C_{0} / 24\right),
$$

and thus the spectral spaces $L_{k}^{1}(\lambda), \lambda<C_{0} / 24$, are of finite $\Gamma$-dimension.

We consider also the operator $k^{-1} \widetilde{\Delta}_{k}^{\prime \prime}$ defined on $L_{n, 0}^{2}\left(\widetilde{M}, \widetilde{E}^{k}\right)$ and denote by $L_{k}^{0}(\lambda)$ its spectral spaces and $N_{\Gamma}^{0}\left(\lambda, k^{-1} \widetilde{\Delta}_{k}^{\prime \prime}\right)$ their $\Gamma$-dimension. Now we can apply Theorem 1.7 for $k^{-1} \widetilde{\Delta}_{k}^{\prime \prime}\left\lceil_{\widetilde{\Omega}}\right.$ on $\widetilde{\Omega}\left(\right.$ with $\left.\widetilde{G}=K_{\widetilde{M}}\right)$. By (1.2) we have $N_{\Gamma}^{0}\left(\lambda, k^{-1} \widetilde{\Delta}_{k}^{\prime \prime}\right) \geqslant N_{\Gamma}^{0}\left(\lambda, k^{-1} \widetilde{\Delta}_{k}^{\prime \prime} \Upsilon_{\Omega}\right)$ and by Theorem 1.7 for $q=0$

(2.8) $\quad \liminf _{k} k^{-n} N_{\Gamma}^{0}\left(\lambda, \frac{1}{k} \widetilde{\Delta}_{k}^{\prime \prime}\right) \geqslant I^{0}(U, \lambda), \quad \lambda<C_{0} / 24, \quad \lambda \in \mathbb{R} \backslash \mathcal{N}$. 
We find now an upper bound. Fix an arbitrary $\delta>0$. Then (2.7) and (1.10) give $\lim \sup _{k} k^{-n} N_{\Gamma}^{1}\left(\lambda, k^{-1} \widetilde{\Delta}_{k}^{\prime \prime}\right) \leqslant I^{1}(U, 12 \lambda+\delta)$. We let $\delta \longrightarrow 0$ :

$$
\underset{k}{\limsup k^{-n} N_{\Gamma}^{1}}\left(\lambda, \frac{1}{k} \widetilde{\Delta}_{k}^{\prime \prime}\right) \leqslant I^{1}(U, 12 \lambda), \quad \lambda<C_{0} / 24 .
$$

We introduce the group $H_{(2)}^{n, 0}\left(\widetilde{M}, \widetilde{E}^{k}\right)=\left\{u \in L_{n, 0}^{2}\left(\widetilde{M}, \widetilde{E}^{k}, \widetilde{\omega}, \widetilde{h}\right): \bar{\partial} u=\right.$ $0\}$ which is a $\Gamma$-module and we find a lower bound for its $\Gamma$-dimension. (We know that the $L^{2}$ norm in degree $(n, 0)$ doesn't actually depend on the metric on $\widetilde{M}$.) Since $\widetilde{\Delta}_{k}^{\prime \prime}$ commutes with $\bar{\partial}$ it follows that the spectral projections of $\widetilde{\Delta}_{k}^{\prime \prime}$ commute with $\bar{\partial}$ too, showing thus $\bar{\partial} L_{k}^{0}(\lambda) \subset L_{k}^{1}(\lambda)$. We have the $\Gamma$-morphism $\bar{\partial}_{\lambda}: L_{k}^{0}(\lambda) \longrightarrow L_{k}^{1}(\lambda)$, the restriction of $\bar{\partial}$ (by the definition of $L_{k}^{0}(\lambda), \bar{\partial}_{\lambda}$ is bounded by $k \lambda$ ). As for usual dimension (see e.g.[14, Lemma 6.11]), $\operatorname{dim}_{\Gamma} \operatorname{ker} \bar{\partial}_{\lambda}+\operatorname{dim}_{\Gamma} \overline{R\left(\bar{\partial}_{\lambda}\right)}=\operatorname{dim}_{\Gamma} L_{k}^{0}(\lambda)$. Moreover $\operatorname{dim}_{\Gamma} \overline{R\left(\bar{\partial}_{\lambda}\right)} \leqslant \operatorname{dim}_{\Gamma} L_{k}^{1}(\lambda)$ and they are finite. Therefore by (2.8) and (2.9), $\operatorname{dim}_{\Gamma} H_{(2)}^{n, 0}\left(M, \widetilde{E}^{k}\right) \geqslant \operatorname{dim}_{\Gamma} \operatorname{ker} \bar{\partial}_{\lambda} \geqslant k^{n}\left[I^{0}(U, \lambda)-I^{1}(U, 12 \lambda)\right]$ for $\lambda<C_{0} / 24$ and $\lambda \in \mathbb{R} \backslash \mathcal{N}$. We can now let $\lambda$ go to zero through these values. The limits $I^{0}(U, 0)$ and $I^{1}(U, 0)$ are calculated in [9, p.224] and identifying the fundamental domain $U$ with $\Omega$, the result is exactly the integral from (2.1).

Remark 2.1. We can obviously apply Theorem 2.1 for the case $M$ compact taking $K=M$ in the hypothesis and $\Omega=M$ in the conclusion. We can work directly with sections in $\widetilde{E}$ rather than $(n, 0)$-forms. More generally, Theorem 2.1 may be applied to generalize the $L^{2}$ Morse inequality of Takayama [27, Main Theorem] to the case of coverings of Zariski open sets in Moishezon manifolds. Also, this theorem may be used to recover [12, Theorem 0.2] of Gromov-Henkin-Shubin already quoted.

To state the following result let us remind that by the definition of Andreotti and Grauert [2] a manifold is called 1-concave if there exists a smooth function $\varphi: X \longrightarrow(a, b]$ where $a \in\{-\infty\} \cup \mathbb{R}, b \in \mathbb{R}$, such that $X_{c}:=\{\varphi>c\} \Subset X$ for all $c \in(a, b]$ and $\varphi$ is strictly plurisubharmonic outside a compact set. Let $E$ be a holomorphic line bundle on $X$. In [21], $[16]$ one constructs $\chi:(-\infty, 0) \longrightarrow \mathbb{R}$ such that $\int_{-1}^{0} \chi(t)^{1 / 2} d t=\infty, \chi^{\prime}(t)^{2} \leqslant$ $4 \chi(t)^{3}, \chi(t) \geqslant 4$ and a hermitian metric $\omega$ which equals $\frac{1}{3} \partial \bar{\partial} \varphi$ near $\partial X_{c}$. For convenience we denote $\psi=c-\varphi$. We define $\omega_{0}=\omega+\chi(\psi) \partial \varphi \wedge \bar{\partial} \varphi$, a complete metric on $X_{c}$ and a hermitian metric $h_{0}=h \exp \left(-A \int_{\inf \psi}^{\psi} \chi(t) d t\right)$ on $E$ over $X_{c}$. 
TheOREm 2.2. Let $X$ be a 1-concave manifold of dimension $n \geqslant 3$ and let $X_{c}$ be a sublevel set such that the exhaustion function $\varphi$ is strictly plurisubharmonic near $\partial X_{c}$. Let $p: \widetilde{X}_{c} \longrightarrow X_{c}$ be a Galois covering of group $\Gamma$. Assume that $\widetilde{X}_{c}$ and $\widetilde{E}$ are endowed with the lifts of the metrics $\omega_{0}$ and $h_{0}$. Then, for $k \longrightarrow \infty$,

$$
\operatorname{dim}_{\Gamma} H_{(2)}^{0}\left(\widetilde{X}_{c}, \widetilde{E}^{k}\right) \geqslant \frac{k^{n}}{n !} \int_{\Omega\left(\leqslant 1, h_{0}\right)}\left(\frac{\imath}{2 \pi} \mathbf{c}\left(E, h_{0}\right)\right)^{n}+o\left(k^{n}\right),
$$

for any sufficiently large open set $\Omega \Subset X_{c}$.

Proof. The metrics $\omega_{0}$ and $h_{0}$ satisfy the following conditions:

(i) Denoting by $\gamma_{i}$ the eigenvalues of $\imath \chi(\psi) \partial \bar{\partial} \psi+\imath \chi^{\prime}(\psi) \partial \psi \wedge \bar{\partial} \psi$ with respect to $\omega_{0}$ we have $\gamma_{1} \leqslant \cdots \leqslant \gamma_{n-1} \leqslant-2 \chi(\psi)$ and $\gamma_{n} \leqslant \chi(\psi)$ so that $\gamma_{n}+\cdots+\gamma_{2} \leqslant(5-2 n) \chi(\psi) \leqslant-\chi(\psi)$ for $n \geqslant 3$ outside a compact set $K:=X_{e} \Subset X_{c}$.

(ii) The torsion operators of the metric $\omega_{0}$ are pointwise bounded by $C_{2} \chi(\psi)^{1 / 2}$ outside $K$.

(iii) The eigenvalues of $\imath \mathbf{c}\left(E, h_{0}\right)$ with respect to $\omega_{0}$ are bounded above on $X_{c}$ by $C_{1}>0$.

Let us take the lifts $\widetilde{\omega}_{0}, \widetilde{h}_{0}$ and $\widetilde{\psi}=c-\varphi \circ p$. It is easy to see that properties (i), (ii) and (iii) are still valid for $\widetilde{\omega}_{0}, \widetilde{h}_{0}$ and $\widetilde{\psi}$ on $\widetilde{X}_{c} \backslash \widetilde{K}$. For $u$ in $\mathcal{D}^{(0,1)}\left(\widetilde{X}_{c} \backslash \widetilde{K}, \widetilde{E}^{k}\right)$ we apply the Bochner-Kodaira-Nakano inequality using the formulas: $\imath \mathbf{c}\left(\widetilde{E}, \widetilde{h}_{0}\right)=\imath \mathbf{c}(\widetilde{E}, \widetilde{h})+\imath A\left(\chi(\widetilde{\psi}) \partial \bar{\partial} \widetilde{\psi}+\chi^{\prime}(\widetilde{\psi}) \partial \widetilde{\psi} \wedge \bar{\partial} \widetilde{\psi}\right)$ and, if $\Theta$ is a real $(1,1)$ form, $\left(\left[\Theta, \widetilde{\Lambda}_{0}\right] u, u\right) \geqslant-\left(\alpha_{n}+\cdots+\alpha_{2}\right)|u|^{2}$, where $\alpha_{1} \leqslant \cdots \leqslant \alpha_{n}$ are the eigenvalues of $\Theta$ with respect to $\widetilde{\omega}_{0}$. We infer $3\left(\widetilde{\Delta}_{k}^{\prime \prime} u, u\right) \geqslant \int\left(-k n C_{1}+k A \chi(\widetilde{\psi})-4 C_{2} \chi(\widetilde{\psi})\right)|u|^{2}$. Since $\chi \geqslant 4$, we derive easily, for large $A$, an estimate analogous to (2.2). From this point the proof of Theorem 2.1 applies with just notational changes.

\section{$\S 3$. Coverings of some strongly pseudoconcave manifolds}

Recall that, by the solution of the Grauert-Riemenschneider conjecture (Siu [24], Demailly [9]), a compact complex manifold $M$ is Moishezon, if it carries a line bundle $E$, which is either semi-positive and positive at one point (Siu's criterion), or (Demailly's criterion) satisfies the inequality:

$$
\int_{M(\leqslant 1)}(\imath \mathbf{c}(E))^{n}>0 .
$$


Then, in fact, $\operatorname{dim} H^{0}\left(M, E^{k}\right) \approx k^{n}$, for large $k$. Our aim is to extend this result in two directions. We allow $M$ to belong to a class of 1-concave manifolds and study their Galois coverings.

If, in the Andreotti-Grauert definition, the function $\varphi$ can be taken such that $a=\inf \varphi=-\infty$, we say that $X$ is hyper 1 -concave ${ }^{1}$. For 1 concave manifolds (which are pseudoconcave in the sense of Andreotti [1]) the transcendence degree of the meromorphic function field is less than or equal to the dimension of $X$. In the latter case we say that the manifold is Moishezon by extending the terminology from compact manifolds.

Let us describe some examples. Let $Y$ be a compact complex manifold, $S$ a complete pluripolar set. Then $M=Y \backslash S$ is hyper 1-concave. Conversely, if $\operatorname{dim} M \geqslant 3$ any hyper 1-concave manifold $M$ is biholomorphic to a complement of a pluripolar set in a compact manifold as a consequence of Rossi's compactification theorem [22]. Another example is $\operatorname{Reg}(X)$ where $X$ is a compact complex space with isolated singularities. Also, if $M$ is a complete Kähler manifold of finite volume and bounded negative sectional curvature, $M$ is hyper 1-concave as shown by Siu-Yau in [25, §1-2]. Moreover, this example falls in the previous case, since by [17, Theorem 0.1] $M$ can be compactified to a projective variety by adding finitely many points.

THEOREM 3.1. Let $M$ be a hyper 1-concave manifold carrying a line bundle $(E, h)$ which is semi-positive outside a compact set. Let $\widetilde{M}$ be a Galois covering of group $\Gamma$ and $\widetilde{E}$ the lifting of $E$. Then, for $k \longrightarrow \infty$

$$
\operatorname{dim}_{\Gamma} H_{(2)}^{n, 0}\left(\widetilde{M}, \widetilde{E}^{k}\right) \geqslant \frac{k^{n}}{n !} \int_{M(\leqslant 1, h)}\left(\frac{\imath}{2 \pi} \mathbf{c}(E, h)\right)^{n}+o\left(k^{n}\right),
$$

where the $L^{2}$ condition is with respect to $\widetilde{h}$ and any metric on $\widetilde{M}$.

Proof. Let us consider a proper function $\varphi: M \longrightarrow(-\infty, 0)$ which is strictly plurisubharmonic outside a compact set. The fact that $\varphi$ goes to $-\infty$ to the ideal boundary of $M$ allows to construct a complete hermitian metric on $M$. Namely we consider the function $\chi=-\log (-\varphi)$ so that $\partial \bar{\partial} \chi=\varphi^{-2} \partial \varphi \wedge \bar{\partial} \varphi-\varphi^{-1} \partial \bar{\partial} \varphi$ which is obviously positive definite on the set where $\partial \bar{\partial} \varphi$ is. We can now patch $\partial \bar{\partial} \chi$ and an arbitrary hermitian metric on $M$ by using a smooth partition of unity to get a metric $\omega_{0}$ on $M$ such

\footnotetext{
${ }^{1}$ Note that not all 1-concave manifolds are hyper 1-concave. Indeed, the complement of $S^{1} \subset \mathbb{C} \subset \mathbb{P}^{1}$ in $\mathbb{P}^{1}$ is 1-concave but cannot possibly be hyper 1-concave since $S^{1}$ is not a polar set in $\mathbb{C}$. I have learnt this example from M. Colţoiu and V. Vâjâitu.
} 
that $\omega_{0}=\partial \bar{\partial} \chi$ on $M \backslash K, K \Subset M$. It is easy to verify that $\omega_{0}$ is complete since $-\chi$ is exhaustive and $\omega_{0}=\omega+\partial(-\chi) \wedge \bar{\partial}(-\chi)$, where $\omega=-\varphi^{-1} \partial \bar{\partial} \varphi$ is a metric on $M \backslash K$, so that $d(-\chi)$ is bounded in the metric $\omega_{0}$. Note that $\omega_{0}$ is obviously Kähler on $M \backslash K$. Let us assume $\imath \mathbf{c}(E, h) \geqslant 0$ on $M \backslash K$ (we stretch $K$ if necessary). We equip $E$ with the metric $h_{\varepsilon}=h \exp (-\varepsilon \chi)$ and the curvature relative to the new metric satisfies $\imath \mathbf{c}\left(E, h_{\varepsilon}\right) \geqslant \varepsilon \omega_{0}$ on $M \backslash K$. We are therefore in the conditions of Theorem 2.1. Since $h_{\varepsilon} \gtrsim h$ there is an injective $\Gamma$-morphism $H_{(2)}^{n, 0}\left(\widetilde{M}, \widetilde{E}^{k}, \widetilde{\omega}_{0}, \widetilde{h}_{\varepsilon}\right) \subset H_{(2)}^{n, 0}\left(\widetilde{M}, \widetilde{E}^{k}, \widetilde{\omega}_{0}, \widetilde{h}\right)$. By this relation and Theorem 2.1 for $H_{(2)}^{n, 0}\left(\widetilde{M}, \widetilde{E}^{k}, \widetilde{\omega}_{0}, \widetilde{h}_{\varepsilon}\right)$,

(3.2) $\liminf _{k} k^{-n} \operatorname{dim}_{\Gamma} H_{(2)}^{n, 0}\left(\widetilde{M}, \widetilde{E}^{k}, \widetilde{\omega}_{0}, \widetilde{h}\right) \geqslant \frac{1}{n !} \int_{\Omega\left(\leqslant 1, h_{\varepsilon}\right)}\left(\frac{\imath}{2 \pi} \mathbf{c}\left(E, h_{\varepsilon}\right)\right)^{n}$

We let now $\varepsilon \searrow 0$ in $(3.1)$; since $h_{\varepsilon}$ converges uniformly together with its derivatives to $h$ on compact sets we see that we can replace $h_{\varepsilon}$ with $h$ in the right-hand side of $(3.1)$. Let $M(q, h)$ be the set where $\imath \mathbf{c}(E, h)$ is non-degenerate and has exactly $q$ negative eigenvalues. Then $M(\leqslant 1, h)=$ $M(0, h) \cup M(1, h)$. By hypothesis $M(1, h) \subset K$ and on $M(0, h)$ the integrand is positive. Hence we can let $\Omega$ exhaust $M$ to get (3.1).

COROLlaRY 3.2. Let $M$ be a hyper 1-concave manifold carrying a line bundle which is semi-positive outside a compact set and satisfies Demailly's condition (D). Then $M$ is Moishezon. In particular the conclusion holds true if $E$ is semi-positive and positive at one point.

Proof. By condition (D) and the previous result for $\Gamma=\{\mathrm{Id}\}$, we have $\operatorname{dim} H^{0}\left(M, E^{k} \otimes K_{M}\right) \geqslant \operatorname{dim} H_{(2)}^{n, 0}\left(M, E^{k}\right) \geqslant C k^{n}$ with $C>0$ and large $k$. We note that the first space is finite dimensional since $M$ is 1-concave. By the Siegel-Serre Lemma, $\operatorname{dim} H^{0}\left(M, E^{k} \otimes K_{M}\right) \leqslant C k^{\varkappa(E)},(k>0)$, (see [16, Proposition 5.7]), where $\varkappa(E)$ is the supremum over $k$ of the generic rank of the canonical meromorphic mapping from $M$ to $\mathbb{P}\left(H^{0}\left(M, E^{k} \otimes K_{M}\right)^{*}\right)$. We obtain $\varkappa(E)=n$, that is, $E^{k} \otimes K_{M}$ gives local coordinates on an open dense set of $M$ for sufficiently large $k$.

Remark 3.1. (a) We can use Corollary 3.2 in the Nadel compactification theorem [18, Theorem 0.1]. Let $M$ be a connected manifold of dimension $\geqslant 3$ satisfying: (i) $M$ is hyper 1-concave, (ii) $M$ is Moishezon, (iii) $M$ can be covered by Zariski-open sets which are uniformized by Stein manifolds. Then $M$ is biholomorphic to $M^{*} \backslash S$ where $M^{*}$ is a compact 
Moishezon space and $S$ is finite. We see thus, that condition (ii) in Nadel's theorem may be replaced with the differential-geometric condition: $M$ possesses a line bundle which is semi-positive outside a compact set and satisfies Demailly's condition (D).

(b) In general, it follows from a theorem of Rossi [22] that, a hyper 1-concave manifold $M$ of dimension $n \geqslant 3$, possessing a semi-positive line bundle satisfying (D), can be compactified so that $M$ is biholomorphic to an open set of a compact Moishezon manifold which is the complement of a complete pluripolar set. We can in fact prove a Kodaira-type characterisation (which holds even for $\operatorname{dim} M=2$ ):

Proposition 3.3. A hyper 1-concave manifold carries a positive line bundle if and only if $M$ is biholomorphic to the complement of a complete pluripolar set in a projective manifold.

Indeed, if $E$ is a positive line bundle, $\imath A \mathbf{c}(E)+\imath \partial \bar{\partial} \chi, A>>0$, is a complete Kähler metric and Hörmander's $L^{2}$ estimates and Andreotti-Tomassini's theorem [4] show that $E$ is ample and $M$ can be embedded in the projective space. By [1], we find a projective compactification of the image of $M$.

Proposition 3.3 and the Rossi example [22] of a non-compactifiable 1concave manifold suggest the following question. Is there a compactification for any hyper 1-concave manifold of dimension 2 ?

(c) Using [18, Theorem 0.2] and the previous remark, we obtain: a manifold $M$ with $\operatorname{dim} M \geqslant 3$ is biholomorphic to the regular part of a complex projective space with isolated singularities if and only if $M$ is hyper 1concave, admits a positive line bundle and has a covering with Zariski open sets uniformized by Stein manifolds.

(d) The argument in the proof of Corollary 3.2 shows that the integral appearing in Theorem 3.1 is finite. Thus, if $E$ is positive outside a compact set $K$ then $M \backslash K$ has finite volume with respect to the metric $\imath \mathbf{c}(E)$ (this observation stems from [17]).

(f) Let $X$ is a compact complex space of dimension $n \geqslant 2$ and with isolated singularities. Suppose that we have a line bundle $E$ on $\operatorname{Reg}(X)$ which is semi-positive in a deleted neighbourhood of $\operatorname{Sing}(X)$ and satisfies $(D)$. Then $X$ is Moishezon. This is a generalization of Takayama's criterion [27] in the case of isolated singularities.

We want now to study the following type of 1-concave manifold. Let $X$ be an irreducible compact complex space with isolated singularities and of 
dimension $\geqslant 2$. We know that $\operatorname{Reg}(X)$ is hyper 1 -concave and we denote by $\varphi: \operatorname{Reg}(X) \longrightarrow \mathbb{R}$ the exhaustion function. Since $\varphi$ is strictly plurisubharmonic outside a compact set we have that the sub-level sets $X_{c}=\{\varphi>c\}$ are 1-concave manifolds i.e. strongly pseudoconcave domains. In our previous paper [16] we have shown that in general if $M$ is a 1-concave manifold of dimension $\geqslant 3$ which carries a hermitian line bundle $E$ which seminegative near the boundary and satisfies (D) then the Kodaira dimension of $E$ is maximal and $M$ is Moishezon. The assumption about the change of curvature sign (i.e. semi-negativity) near the boundary is imposed by the construction of complete hermitian metrics $\omega_{0}$ and $h_{0}$ as in Theorem 2.2 which give the $L^{2}$ estimate needed and preserve condition (D) for $h_{0}$. The restriction on dimension comes from the fact that we need an $L^{2}$ estimate in bi-degree $(0,1)$.

Of course, usually we are given an overall positive bundle $E$ on $M$. We show that for manifolds $X_{c}$ as before we can also apply the criterion in [16] alluded to by modifying the metric. Let us consider a covering $\left\{U_{\alpha}\right\}$ of $X$ and embeddings $\iota_{\alpha}: U_{\alpha} \hookrightarrow \mathbb{C}^{N_{\alpha}}$ such that $\left.E\right|_{U_{\alpha}}$ is the inverse image by $\iota_{\alpha}$ of the trivial line bundle $\mathbb{C}_{\alpha}$ on $\mathbb{C}^{N_{\alpha}}$. Moreover we consider hermitian metrics $h_{\alpha}=e^{-\varphi_{\alpha}}$ on $\mathbb{C}_{\alpha}$ such that $\iota_{\alpha}^{*} h_{\alpha}=\iota_{\beta}^{*} h_{\beta}$ on $U_{\alpha} \cap U_{\beta} \cap \operatorname{Reg}(X)$. The system $h=\left\{\iota_{\alpha}^{*} h_{\alpha}\right\}$ is called a hermitian metric on $E$ over $X$. It clearly induces a hermitian metric on $E$ over $\operatorname{Reg}(X)$.

THEOREM 3.4. Let $X$ be an irreducible compact complex space with isolated singularities and let $X_{c}$ be the sublevel sets of the hyper 1-concave manifold $\operatorname{Reg}(X)$. Assume that there exists a holomorphic line bundle $E \longrightarrow X$ with a smooth hermitian metric such that condition (D) is fulfilled on $\operatorname{Reg}(X)$. Then for sufficiently small $c$ there exists a metric on $E$ such that $E$ is negative in the neighbourhood of $\partial X_{c}$ and $\int_{X_{c}(\leqslant 1)}(\imath \mathbf{c}(E))^{n}>0$.

Proof. Let $\pi: \bar{X} \longrightarrow X$ be a resolution of singularities of $X$. Let us denote by $D_{i}$ the components of the exceptional divisor. Then there exist positive integers $n_{i}$ such that $D:=\sum n_{i} D_{i}$ admits a smooth hermitian metric such that the induced line bundle $[D]$ is negative in a neighbourhood $\widetilde{U}$ of $D$ (cf. [23]). Let us consider a canonical section $s$ of $[D]$, i.e. $D=(s)$, and denote by $|s|^{2}$ the pointwise norm of $s$ with respect to the above metric. By Lelong-Poincaré equation $\varphi=\log |s|^{2}$ is strictly plurisubharmonic on $\widetilde{U} \backslash D$. By using a smooth function on $\bar{X}$ with compact support in $\widetilde{U}$ which equals one near $D$ we construct a smooth function $\chi$ on $\bar{X} \backslash D \simeq \operatorname{Reg}(X)$ 
such that $\chi=-\log \left(-\log |s|^{2}\right)$ on $\widetilde{U} \backslash D$. Since $\log |s|^{2}$ goes to $-\infty$ on $D$, this is the analogue of the function constructed in the proof of Theorem 3.1. As there we show that $\imath \partial \chi \wedge \bar{\partial} \chi \leqslant \imath \partial \bar{\partial} \chi$. Let us consider a metric $\omega$ on $\operatorname{Reg}(X)$ which on every open set $U_{\alpha}$ as above is the pullback of a hermitian metric on the ambient space $\mathbb{C}^{N_{\alpha}}, \omega=\iota_{\alpha}^{*} \omega_{\alpha}$. Since the singularities are isolated we may assume that the metric is distinguished, that is, in the neighbourhood of the singular points $\omega_{\alpha}$ is the euclidean metric. We consider then the metric (Kähler near Sing $(X)) \omega_{0}=A \omega+\partial \bar{\partial} \chi$ where $A>0$ is chosen sufficiently large (to ensure that $\omega_{0}$ is a metric away from the open set where $\partial \bar{\partial} \chi$ is positive definite). It is easily seen that $\omega_{0}$ is complete by the same argument as in the proof of Theorem 3.1. This kind of metrics were introduced by Saper in [23]. They have finite volume.

Let us consider now a neighbourhood $U$ of the singular set. We assume that $U$ is small enough so that there are well defined on $U$ a potential $\rho$ for $\omega$ and a potential $\eta$ for the curvature $\imath \mathbf{c}(E)$ (they are restrictions from ambient spaces). By suitably cutting-off we may define a function $\psi \in \mathcal{C}^{\infty}(\operatorname{Reg}(X))$ such that $\psi=-\chi-\eta-A \rho$ near $\operatorname{Sing}(X)$. Since the potentials $\rho$ and $\eta$ are smooth, they are bounded so that $\psi$ tends to $\infty$ at the singular set $\operatorname{Sing}(X)$. Let us consider a smooth function $\gamma: \mathbb{R} \longrightarrow \mathbb{R}$ such that $\gamma(t)=0$ if $t \leqslant 0$, $\gamma(t)=t$ if $t \geqslant 1$ and the functions $\gamma_{\nu}: \mathbb{R} \longrightarrow \mathbb{R}$ given by $\gamma_{\nu}(t)=\gamma(t-\nu)$ for all positive integers $\nu$. Let us denote the hermitian metric on $E$ by $h$ and let us consider the following metric on $E: h_{\nu}=h \exp \left(-\gamma_{\nu}(\psi)\right)$, with curvature $\imath \mathbf{c}\left(E, h_{\nu}\right)=\imath \mathbf{c}(E, h)+\imath \gamma_{\nu}^{\prime}(\psi) \partial \bar{\partial} \psi+\imath \gamma_{\nu}^{\prime \prime}(\psi) \partial \psi \wedge \bar{\partial} \psi$. On the set $\{\psi \geqslant \nu+1\}$ we have $\gamma_{\nu}(\psi)=\psi-\nu$ so that $\gamma_{\nu}^{\prime}(\psi)=1$ and $\gamma_{\nu}^{\prime \prime}(\psi)=0$ and therefore $\imath \mathbf{c}\left(E, h_{\nu}\right)=\imath \mathbf{c}(E, h)+\imath \partial \bar{\partial} \psi$. Since $\psi$ goes to $\infty$ when we approach the singular set we may choose $\nu_{0}$ such that for $\nu \geqslant \nu_{0}$ we have $\{\psi \geqslant \nu+1\} \subset U$ where $U$ is a sufficiently small neighbourhood of $\operatorname{Sing}(X)$. Bearing in mind the meaning of $\eta$ and $\rho$ together with the definition of $\omega_{0}$ it is straightforward that $\imath \mathbf{c}\left(E, h_{\nu}\right)=-\omega_{0}$ on $\{\psi \geqslant \nu+1\}$, that is $\left(E, h_{\nu}\right)$ is negative on this set. We denote $\Omega_{\nu}$ the compact set $\{\psi \leqslant \nu+2\}$. We decompose this set in $\Omega_{\nu}^{\prime}=\{\psi \leqslant \nu\}$ and $\Omega_{\nu}^{\prime \prime}=\{\nu \leqslant \psi \leqslant \nu+2\}$. On $\Omega_{\nu}^{\prime}$ we have $\gamma_{\nu}(\psi)=0$ and $\imath \mathbf{c}\left(E, h_{\nu}\right)=\imath \mathbf{c}(E, h)$. We infer that

$$
\int_{\Omega_{\nu}^{\prime}\left(\leqslant 1, h_{\nu}\right)}\left(\imath \mathbf{c}\left(E, h_{\nu}\right)\right)^{n}=\int_{\operatorname{Reg}(X)(\leqslant 1, h)} \mathbf{1}_{\Omega_{\nu}^{\prime} \alpha_{1}} \cdots \alpha_{n} d V_{0}
$$

where $\alpha_{1}, \cdots, \alpha_{n}$ are the eigenvalues of $\imath \mathbf{c}(E, h)$ with respect to $\omega_{0}$ and $d V_{0}=\omega_{0}^{n} / n$ !. Since $\imath \mathbf{c}(E, h)$ is dominated by the euclidean metric near $\operatorname{Sing}(X), \imath \mathbf{c}(E, h)$ is dominated by $\omega$ and by $\omega_{0}$. Hence the product $\alpha_{1} \cdots \alpha_{n}$ 
is bounded on $\operatorname{Reg}(X)$. Since $\operatorname{Reg}(X)(\leqslant 1)$ has finite volume with respect to $\omega_{0}$ the functions $\left|\mathbf{1}_{\Omega_{\nu}^{\prime}} \alpha_{1} \cdots \alpha_{n}\right|$ are bounded by an integrable function. On the other hand $\mathbf{1}_{\Omega_{\nu}^{\prime}} \longrightarrow 1$ when $\nu \longrightarrow \infty$ so that the integrals in (4.3) tend to $\int_{\operatorname{Reg}(X)(\leqslant 1, h)}(\imath \mathbf{c}(E, h))^{n}$ which is assumed to be positive. Thus it suffices to show that the integral on the set $\Omega_{\nu}^{\prime \prime}$ i.e. $\int_{\Omega_{\nu}^{\prime \prime}\left(\leqslant 1, h_{\nu}\right)}\left(\imath \mathbf{c}\left(E, h_{\nu}\right)\right)^{n}$ tends to zero as $\nu \longrightarrow \infty$. For this purpose we use the obvious bound $\int_{\Omega_{\nu}^{\prime \prime}\left(\leqslant 1, h_{\nu}\right)}\left(\imath \mathbf{c}\left(E, h_{\nu}\right)\right)^{n} \leqslant \sup \left|\delta_{1} \cdots \delta_{n}\right| \cdot \operatorname{vol}\left(\Omega_{\nu}^{\prime \prime}\right)$ where $\delta_{1}, \cdots, \delta_{n}$ are the eigenvalues of $\imath \mathbf{c}\left(E, h_{\nu}\right)$ with respect to $\omega_{0}$ and the volume is taken in the same metric. We use now the minimum-maximum principle to see that (i) $\delta_{1}$ is bounded below and $\delta_{2}, \cdots, \delta_{n}$ are bounded above on the set of integration $\Omega_{\nu}^{\prime \prime}\left(1, h_{\nu}\right)$ and (ii) $\delta_{1}, \cdots, \delta_{n}$ are upper bounded on $\Omega_{\nu}^{\prime \prime}\left(0, h_{\nu}\right)$. For this we need the domination of $\imath \mathbf{c}(E, h)$ by $\omega$ and the boundedness of $\gamma_{\nu}^{\prime}$ and $\gamma_{\nu}^{\prime \prime}$. Since $\operatorname{vol}\left(\Omega_{\nu}^{\prime \prime}\right) \longrightarrow 0$ as $\nu \longrightarrow \infty$ our contention follows. Hence for large $\nu$ the metric $h_{\nu}$ does the required job.

Since the manifold $\bar{X}_{c}$ is compact Theorem 3.4 can be used to prove stability results for perturbation of the complex structure of $\bar{X}_{c}$. Since our approach relies on the use of a sufficiently positive line bundle $E$ we need to consider perturbations of the complex structure which lift to a perturbation of $E$. This kind of situation was studied by L. Lempert in [15, Lemma 4.1].

Corollary 3.5. Let $X$ be a Moishezon variety with isolated singularities and dimension $n \geqslant 3$. Let $\mathcal{J}$ denote the complex structure of $\operatorname{Reg}(X)$. Let $E=[Z]$, where $Z \subset \operatorname{Reg}(X)$ is a compact non-singular hypersurface. Assume that $E$ satisfies (D). Then for sufficiently small $c$ and any complex structure $\mathcal{J}^{\prime}$ on $\bar{X}_{c}$ such that (1) $\mathcal{J}^{\prime}$ is sufficiently close to $\mathcal{J}$ in the $\mathcal{C}^{\infty}$ topology, and (2) $T(Z)$ is $\mathcal{J}^{\prime}$ invariant, there exists a $\mathcal{J}^{\prime}$-holomorphic line bundle $E^{\prime}$ on $\bar{X}_{c}$ which is negative near $b X_{c}$ and satisfies $(\mathrm{D})$. In particular $\left(\bar{X}_{c}, \mathcal{J}^{\prime}\right)$ is a Moishezon pseudoconcave manifold and any compactification of $\left(\bar{X}_{c}, \mathcal{J}^{\prime}\right)$ is Moishezon.

Proof. Let us first choose $c_{0}$ such that for $c<c_{0}$ there exists a 'good' hermitian metric $h$ on $E$ over a neighbourhood of $X_{c}$ as in Theorem 3.4. We use now the description of the lifting of $\mathcal{J}^{\prime}$ with properties (1) and (2) as given in [15]. Namely, $Z$ determines a new $\mathcal{J}^{\prime}$ holomorphic line bundle $E^{\prime} \longrightarrow\left(\bar{X}_{c}, \mathcal{J}^{\prime}\right)$. There exists a finite open covering $\mathcal{U}=\{U\}$ of $\bar{X}_{c}$ such that $E$ and $E^{\prime}$ are trivial on each $U$ and they are defined by multiplicative cocycles $\left\{g_{U V}\right\}$ where $g_{U V}$ is $\mathcal{J}$-holomorphic on $\bar{U} \cap \bar{V}$ and $\left\{g_{U V}^{\prime}\right\}$ where $g_{U V}^{\prime}$ is $\mathcal{J}^{\prime}$-holomorphic on $\bar{U} \cap \bar{V}$ for $U, V \in \mathcal{U}$. Moreover $g_{U V}$ and $g_{U V}^{\prime}$ 
are as close as we please assuming $\mathcal{J}$ and $\mathcal{J}^{\prime}$ are sufficiently close. (By 'close' we always understand close in the $\mathcal{C}^{\infty}$ topology.) Next we can define a smooth bundle isomorphism $E \longrightarrow E^{\prime}$ by resolving the smooth additive cocycle $\log \left(g_{U V}^{\prime} / g_{U V}\right)$ in order to find smooth functions $f_{U}$, close to 1 on a neighbourhood of $\bar{U}$ such that $g_{U V}^{\prime}=f_{U} g_{U V} f_{V}^{-1}$. Then the isomorphism between $E$ and $E^{\prime}$ is given by $f=\left\{f_{U}\right\}$. The metric $h$ is given in terms of the covering $\mathcal{U}$ by a collection $h=\left\{h_{U}\right\}$ of smooth strictly positive functions satisfying the relation $h_{V}=h_{U}\left|g_{U V}\right|$. We define a hermitian metric $h^{\prime}=\left\{h_{U}^{\prime}\right\}$ on $E^{\prime}$ by $h_{U}^{\prime}=h_{U}\left|f_{U}^{-1}\right| ; h_{U}^{\prime}$ is close to $h_{U}$. The curvature form of $E^{\prime}$ is given by $\frac{\imath}{2 \pi} \mathbf{c}\left(E^{\prime}\right)=\frac{1}{4 \pi} d \circ \mathcal{J}^{\prime} \circ d\left(\log h_{U}^{\prime}\right)$. Therefore, when $\mathcal{J}^{\prime}$ is sufficiently close to $\mathcal{J}, \frac{\imath}{2 \pi} \mathbf{c}\left(E^{\prime}\right)$ is negative near the boundary of $\bar{X}_{c}$ and, since the eigenvalues of $\frac{\imath}{2 \pi} \mathbf{c}\left(E^{\prime}\right)$ are close to those of $\frac{\imath}{2 \pi} \mathbf{c}(E), E^{\prime}$ satisfy the condition (D) i.e. $\int_{X_{c}(\leqslant 1)}\left(\imath \mathbf{c}\left(E^{\prime}\right)\right)^{n}>0$. We can apply thus [16, Corollary $4.3]$ to the strongly pseudoconcave manifold $\left(\bar{X}_{c}, \mathcal{J}^{\prime}\right)$ to conclude.

Remark 3.2. If $[Z]$ is positive, part of the stability property follows from the rigidity of embeddings with positive normal bundle. Indeed, assume $N_{Z}=[Z] \Upsilon_{Z}$ is positive in $\left(\bar{X}_{c}, \mathcal{J}^{\prime}\right.$ ) (for any $c$ such that this manifold is still pseudoconcave). Then Ph. Griffiths [10, p.387] has shown that there exists a neighbourhood $W$ of $Z$ such that the mapping $\Phi:\left(X_{c}, \mathcal{J}^{\prime}\right)--\rightarrow \mathbb{P}^{N}$ given by $[m Z]$ is an embedding of $W$ for large $m$. Thus $\left(X_{c}, \mathcal{J}^{\prime}\right)$ is Moishezon. Our result deals with the slightly more general situation of a 'big' embedding i.e. when $[Z]$ is not ample but satisfies condition (D). Moreover we have a useful quantitative way of measuring whether the perturbed structure is Moishezon.

Corollary 3.6. Let $\left(\bar{X}_{c}, \mathcal{J}^{\prime}\right)$ and $E^{\prime}$ be as in Corollary 3.5. Then there exists hermitian metrics on $X_{c}$ and $E^{\prime}$ and a positive constant $C$ such that for any Galois covering $\tilde{X}_{c} \longrightarrow X_{c}$ of group $\Gamma$, for $k \longrightarrow \infty$ : $\operatorname{dim}_{\Gamma} H_{(2)}^{0}\left(\widetilde{X}_{c}, \widetilde{E}^{k}\right) \geqslant C k^{n}$, the $L^{2}$ condition being with respect to lifts of the hermitian metrics on $X_{c}$ and $E^{\prime}$.

Proof. We know that we have a metric $h$ on $E^{\prime}$ satisfying the conclusion of Theorem 3.4. Then, as in Theorem 2.2, we can construct metrics $\omega_{0}$ and $h_{0}$ in order to obtain (2.10). Note that the integral in (2.10) depends on the modified metric $h_{0}$ so we cannot always infer that it is positive even if $\left(E^{\prime}, h\right)$ satisfies (D). But under the assumption of semi-negativity of $h$ near the boundary we can construct an $h_{0}$ such that the integral in (2.10) is positive (cf. Corollary 4.3 of [16]). Thus we conclude by Theorem 2.2. 


\section{$\S 4$. Weak Lefschetz theorems}

Nori [20] generalized the Lefschetz hypersurface theorem. Assume $X$ and $Y$ are smooth connected projective manifolds and $Y$ is a hypersurface in $X$ with positive normal bundle and $\operatorname{dim} Y \geqslant 1$. Then the image of $\pi_{1}(Y)$ in $\pi_{1}(X)$ is of finite index. Recently, Napier and Ramachandran [19] proposed an analytic approach and generalized Nori's theorem showing that $Y$ may have arbitrary codimension (but $\operatorname{dim} Y \geqslant 1$ ). They use the $\bar{\partial}$-method on complete Kähler manifolds to separate the sheets of appropriate coverings. In the sequel we use the Morse inequalities to study non-necessarily Kähler manifolds. First we introduce the notion of formal completion (see [7]). Let $Y$ be a complex analytic subspace of the manifold $U$ and denote by $\mathcal{I}_{Y}$ the ideal sheaf of $Y$. The formal completion $\widehat{U}$ of $U$ with respect to $Y$ is the ringed space $\left(\widehat{U}, \mathcal{O}_{\widehat{U}}\right)=\left(Y\right.$, proj $\left.\lim \mathcal{O}_{U} / \mathcal{I}_{Y}^{\nu}\right)$. If $\mathcal{F}$ is an analytic sheaf on $U$ we denote by $\widehat{\mathcal{F}}$ the sheaf $\widehat{\mathcal{F}}=\operatorname{proj} \lim \mathcal{F} \otimes\left(\mathcal{O} / \mathcal{I}_{Y}^{\nu}\right)$. If $\mathcal{F}$ is coherent then $\widehat{\mathcal{F}}$ is too. Moreover by [7, Proposition VI.2.7] the kernel of the mapping $H^{0}(U, \mathcal{F}) \longrightarrow H^{0}(\widehat{U}, \widehat{\mathcal{F}})$ consists of the sections of $\mathcal{F}$ which vanish on a neighbourhood of $Y$. Hence for locally free $\mathcal{F}$ the map is injective.

THEOREM 4.1. Let $M$ be a manifold and $E \longrightarrow M$ be a line bundle in one of the situations of Theorem 2.1, 2.2 or 3.1 and assume that the integral in (2.1), (2.10) or (3.1) respectively, is positive. Let moreover $Y$ be a connected compact complex subspace of $M$ satisfying: (i) for any $k$, $\operatorname{dim} H^{0}\left(\widehat{M}, \widehat{\mathcal{F}}_{k}\right)<\infty$, where $\mathcal{F}_{k}=\mathcal{O}\left(E^{k} \otimes K_{M}\right)$, (ii) the image $G$ of $\pi_{1}(Y)$ in $\pi_{1}(M)$ is normal in $\pi_{1}(M)$. Then $G$ is of finite index in $\pi_{1}(M)$.

Proof. We follow the proof given in [19]. Since $G$ is normal there exists a connected Galois covering $\pi: \widetilde{M} \longrightarrow M$ such that the group of deck transformations is $\Gamma=\pi_{1}(M) / G$. The cardinal $|\Gamma|$ equals the index of $G$ in $\pi_{1}(M)$. Let $\widetilde{E}=\pi^{-1} E$. By applying, after case, Theorem 2.1, 2.2 or 3.1, there exists $C>0$ such that for large $k, \operatorname{dim}_{\Gamma} H_{(2)}^{n, 0}\left(\widetilde{M}, \widetilde{E}^{k}\right) \geqslant C k^{n}$. (Remark that Theorem 2.2 is also valid if we twist $\widetilde{E}^{k}$ with any $\Gamma$-invariant bundle.) Let us choose a small open neighbourhood $V$ of $Y$ such that $\pi_{1}(Y) \longrightarrow$ $\pi_{1}(V)$ is an isomorphism; so the image of $\pi_{1}(V)$ in $\pi_{1}(M)$ is $G$. Hence, if we denote by $\jmath$ the inclusion of $V$ in $M$, there exists a holomorphic lifting $\widetilde{\jmath}: V \longrightarrow \widetilde{M}, \pi \circ \widetilde{\jmath}=\jmath$. Since $\widetilde{\jmath}$ is locally biholomorphic the pull-back $\operatorname{map} \widetilde{\jmath}^{*}: H_{(2)}^{n, 0}\left(\widetilde{M}, \widetilde{E}^{k}\right) \longrightarrow H^{n, 0}\left(V, E^{k}\right)$ is injective. On the other hand $H^{0}\left(V, \mathcal{F}_{k}\right) \hookrightarrow H^{0}\left(\widehat{V}, \widehat{\mathcal{F}}_{k}\right)=H^{0}\left(\widehat{M}, \widehat{\mathcal{F}}_{k}\right)$. By (i) the latter space is finite 
dimensional so $\operatorname{dim} H_{(2)}^{n, 0}\left(\widetilde{M}, \widetilde{E}^{k}\right)<\infty$. But $\operatorname{dim}_{\Gamma} H_{(2)}^{0}\left(\widetilde{M}, \widetilde{E}^{k} \otimes K_{\widetilde{M}}\right)>0$ for $k>C^{-1 / n}$. If $\Gamma$ were infinite this would yield $\operatorname{dim} H_{(2)}^{n, 0}\left(\widetilde{M}, \widetilde{E}^{k}\right)=\infty$, a contradiction. Therefore $|\Gamma|<\infty$ and $\operatorname{dim} H_{(2)}^{n, 0}\left(\widetilde{M}, \widetilde{E}^{k}\right) \geqslant C|\Gamma| k^{n} \geqslant|\Gamma|$ for $k>C^{-1 / n}$. Thus $|\Gamma| \leqslant \operatorname{dim} H^{0}\left(\widehat{M}, \widehat{\mathcal{F}}_{k}\right)$ for large $k$.

Remark 4.1. (a) By a theorem of Grothendieck [11], condition (i) is fulfilled if $Y$ is locally a complete intersection with ample normal bundle $N_{Y}$ (or $k$-ample in the sense of Sommese, $k=\operatorname{dim} Y-1$ ).

(b) We can replace condition (i) with the requirement that $Y$ has a fundamental system of pseudoconcave neighbourhoods $\{V\}$. Then $\operatorname{dim} H^{0}\left(V, \mathcal{F}_{k}\right)$ is finite by [1]. This happens, for example, when $Y$ is a smooth hypersurface and $N_{Y}$ has at least one positive eigenvalue, or, if $Y$ has arbitrary codimension, when $N_{Y}$ is sufficiently positive in the sense of Griffiths [10, Proposition 8.2].

(c) Condition (ii) is trivially satisfied if $\pi_{1}(Y)=0$. Thus, if $M$ contains a simply connected subvariety satisfying either (a) or (b), $\pi_{1}(M)$ is finite.

(d) It follows from Remark 2.1 that, Theorem 4.1 holds for compact manifolds $M$, and also for Zariski open sets in Moishezon manifolds.

(f) By Corollary 3.6, Theorem 4.1 can be applied to the perturbed structures considered there.

Acknowledgements. We want to express our wholehearted thanks the following people: V. Iftimie for initiating this project, J. Kollár, G. Henkin, L. Lempert for useful conversations, J. Leiterer for hospitality at Berlin and, last but not least, the referee for suggestions concerning the terminology and presentation.

\section{REFERENCES}

[1] A. Andreotti, Théorèmes de dépendance algébrique sur les espaces complexes pseudo-concaves, Bull. Soc. Math. France, 91 (1963), 1-38.

[2] A. Andreotti, H. Grauert, Théorème de finitude pour la cohomologie des espaces complexes, Bull. Soc. Math. France, 90 (1962), 193-259.

[3] A. Andreotti, E. Vesentini, Carleman estimates for the Laplace-Beltrami equation on complex manifolds, Inst. Hautes Etudes Sci. Publ. Math., 25 (1965), 81-130.

[4] A. Andreotti, G. Tomassini, Some remarks on pseudoconcave manifolds, Essays in Topology and Related Topics, dedicated to G. de Rham (R. Narasimhan, A. Haefliger, eds.), Springer, Berlin-Heidelberg-New York (1970), pp. 84-105. 
[5] M. F. Atiyah, Elliptic operators, discrete groups and von Neumann algebras, Astérisque, 32-33 (1976), pp. 43-72.

[6] T. Bouche, Inégalités de Morse pour la $d^{\prime \prime}$-cohomologie sur une variété noncompacte, Ann. Sci. Ecole Norm. Sup., 22 (1989), 501-513.

[7] C. Bănică, O. Stănăşilă, Algebraic methods in the global theory of complex spaces, Wiley, New York, 1976.

[8] H.L. Cycon, R.G. Froese, W. Kirsch, B. Simon, Schrödinger operators with applications to quantum physics, Texts and Monographs in Physics, Springer-Verlag, 1987.

[9] J. P. Demailly, Champs magnétiques et inégalités de Morse pour la $d^{\prime \prime}$-cohomologie, Ann. Inst. Fourier, 35 (1985), 189-229.

[10] $\mathrm{Ph}$. A. Griffiths, The extension problem in complex analysis; embedding with positive normal bundle, Amer. J. Math., 88 (1966), 366-446.

[11] A. Grothendieck, Cohomologie locale des faisceaux cohérents et théorèmes de Lefschetz locaux et globaux, North-Holland, Amsterdam, 1968.

[12] M. Gromov, M. G. Henkin, M. Shubin, $L^{2}$ holomorphic functions on pseudo-convex coverings, GAFA, 8 (1998), 552-585.

[13] G.Henniart, Les inégalités de Morse (d'après Witten), Astérisque, 1983/84 (1985), no. 121/122, 43-61.

[14] J. Kollár, Shafarevich maps and automorphic forms, Princeton University Press, Princeton, NJ, 1995.

[15] L. Lempert, Embeddings of three dimensional Cauchy-Riemann manifolds, Math. Ann., 300 (1994), 1-15.

[16] G. Marinescu, Asymptotic Morse Inequalities for Pseudoconcave Manifolds, Ann. Scuola Norm. Sup. Pisa Cl. Sci. (4), 23 (1996), no. 1, 27-55.

[17] A. Nadel, H. Tsuji, Compactification of complete Kähler manifolds of negative Ricci curvature, J. Differential Geom., 28 (1988), no. 3, 503-512.

[18] A. Nadel, On complex manifolds which can be compactified by adding finitely many points, Invent. Math., 101 (1990), no. 1, 173-189.

[19] T. Napier, M. Ramachandran, The $L^{2}$-method, weak Lefschetz theorems and the topology of Kähler manifolds, JAMS, 11, no. 2, 375-396.

[20] M.V. Nori, Zariski's conjecture and related problems, Ann. Sci. Ec. Norm. Sup., 16 (1983), 305-344.

[21] T. Ohsawa, Isomorphism theorems for cohomology groups of weakly 1-complete manifolds, Publ. Res. Inst. Math. Sci., 18 (1982), 191-232.

[22] H. Rossi, Attaching analytic spaces to an analytic space along a pseudoconcave boundary, Proceedings of the Conference on Complex Analysis (Minneapolis 1964), Springer-Verlag, Berlin (1965), pp. 242-256.

[23] L. Saper, $L^{2}$-cohomology and intersection homology of certain algebraic varieties with isolated singularities, Invent. Math., 82 (1985), no. 2, 207-255.

[24] Y. T. Siu, A vanishing theorem for semipositive line bundles over non-Kähler manifolds, J. Diff. Geom., 19 (1984), 431-452.

[25] Y. T. Siu, S. T. Yau, Compactification of negatively curved complete Kähler manifolds of finite volume, Ann. Math. Stud., 102 (1982), 363-380. 
[26] M. Shubin, Semiclassical asymptotics on covering manifolds and Morse inequalities, GAFA, 6 (1996), no. 2, 370-409.

[27] S. Takayama, A differential geometric property of big line bundles, Tôhoku Math. J. (2), 46 (1994), no. 2, 281-291.

[28] E. Witten, Supersymmetry and Morse theory, J. Diff. Geom., 17 (1982), 661-692.

Radu Todor

Faculty of Mathematics, University of Bucharest

Str Academiei 14, Bucharest

Romania

Ionut Chiose

Department of Mathematics

SUNY at Stony Brook, Stony Brook, NY 11794-3651

$U S A$

chiose@math.sunysb.edu

George Marinescu

Institute of Mathematics of the Romanian Academy, Bucharest

Romania

CurRent AdDress

Institut für Mathematik, Humboldt-Universität zu Berlin, Unter den Linden 6, 10099 Berlin

Deutschland

george@ mathematik.hu-berlin.de 